\title{
Numerical Analysis of the Incident ion Energy and Angle Distribution in the DC Magnetron Sputtering for the Variation of Gas Pressure
}

\author{
Min Young Hur a, Sehun Oha ${ }^{\mathrm{a}}$, Ho Jun Kim ${ }^{\mathrm{b}}$, and Hae June Lee ${ }^{\mathrm{a}}$ \\ ${ }^{a}$ Department of Electrical and Computer Engineering, Pusan National University, Busan 46241, South Korea \\ ${ }^{b}$ Memory Thin Film Technology Team, Samsung Electronics, Hwaseong 18382, South Korea
}

Received January 16, 2018; revised January 29, 2018; accepted January 30, 2018

\begin{abstract}
The ion energy and angle distributions (IEADs) in the DC magnetron sputtering systems are investigated for the variation of gas pressure using particle-in-cell simulation. Even for the condition of collisionless ion sheath at low pressure, it is possible to change the IEAD significantly with the change of gas pressure. The bombarding ions to the target with low energy and large incident angle are observed at low pressure when the sheath voltage drop is low. It is because the electron transport is hindered by the magnetic field at low pressure because of few collisions per electron gyromotion while the ions are not magnetized. Therefore, the space charge effect is the most dominant factor for the determination of IEADs in low-pressure magnetron sputtering discharges.
\end{abstract}

Keywords: Magnetron sputtering, Particle-in-cell simulation, Ion energy distribution

\section{Introduction}

The magnetron sputtering (MS) is widely used for the deposition of a wide range of industrially important coating because of good homogeneity and quality even at low deposition temperature [1-6]. The experimental measurement is helpful for understanding the mechanism of MS [7-10]. For the deep understating, however, it is necessary to analyze the system using computer simulation. The sputtered target profile is dominantly affected by the ion energy and angle distributions (IEADs), and thus it is necessary to control the IEADs to extend the use of targets and to enhance the deposited film quality. In this study, we report the investigation of ion energy and angle distribution of MS analyzed by particle-in-cell (PIC) simulation [11].

The PIC method has the advantage of high accuracy compared with the fluid model. However, it suffers from drawbacks such as large computation load and difficulty of multi-species and many reactions. Nonetheless, the PIC method is widely used in the simulation of MS [12-17]. Because the MS does not require the multi-species and many reactions due to the background gas is simple in most cases, and large computation load can be overcome by parallel computing such as general purpose graphics processing unit (GPGPU) [18]. The advantage of the PIC method in MS is that the ion bombardment information to

*Corresponding author

E-mail: haejune@pusan.ac.kr the target can be obtained accurately such as ion energy, ion incident angle, and sputtering yield.

In this study, the optimized parallel PIC with the OpenMP is used for magnetron simulation. The simulation method and the simulation condition are explained in Sec. 2 followed by the simulation results for the variation of the gas pressure in Sec. 3. Finally, the conclusion is presented in Sec. 4.

\section{Numerical Modeling}

\section{Simulation Method}

The MS system is simulated using the PIC method in the electrostatic and magnetostatic system. The electric potential is calculated by solving the Poisson equation. In this study, it is assumed that the induced magnetic field by plasma motion is much smaller than the static magnetic field by magnets. The static magnetic field is calculated using a freeware, FEMM (Finite Element Method Magnetics). The electric field and magnetic field push the charged particle using Boris method [19]. The collisions between the neutral particles and charged particles are calculated by Monte Carlo method using null collisions [20].

The sputtering yield has been studied theoretically, experimentally, and numerically in many types of research [21-24]. In this system, the sputtering yield is calculated using Yamamura's formula [24] which is a fitting equation depends on the incident ion energy and angle according to 


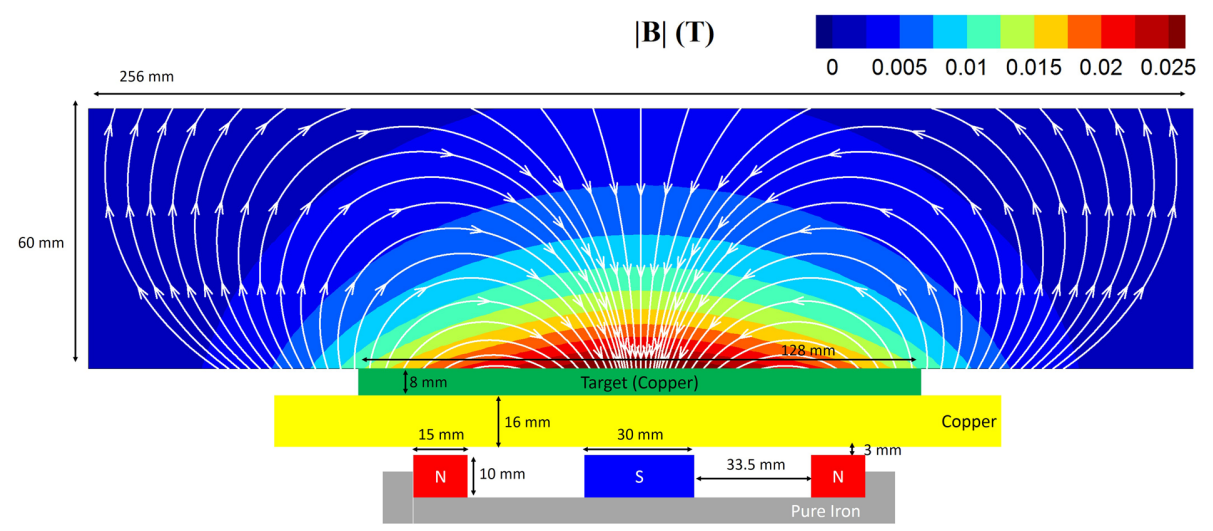

Figure 1. The DC MS system considered in this study with the intensity and flux line of magnetic field.

the target species and ion species.

\section{Simulation conditions}

Figure 1 shows the simulation domain. It shows the amplitude of magnetic field and magnetic flux line. The maximum value of magnetic flux density is about 250 Gauss on the target surface. The number of cells is 512 by 120 and the time step is $10^{-11} \mathrm{~s}$. The number ratio of real particles to a computation particle is $10^{8}$. The considered pressure is $5,10,15$, and 20 mTorr of argon gas. The simulations are conducted on a current-driven mode with $0.5 \mathrm{~A}$. The left and the right boundaries are applied to periodic boundary condition. The current-driven mode assumes the open circuit [25] with a fixed source current. The source current is the sum of the displacement current and convection current. The convection current is calculated by collecting the charged particle from the target electrode. Therefore the displacement current is controlled to fix the source current. The displacement current is related to the potential of the target electrode. If the simulation is saturated, the potential of the target electrode is also saturated. It means that the displacement current becomes zero. Then, the source current is the same as the convection current. The convection current consists of the electron current and the ion current. In the DC MS system, the electron current is much smaller than the ion current because of the magnetic field and the negative electrode potential. Therefore, the source current is the same as the ion convection current,which means that the number of bombarding ion to the target depends on only the source current.

\section{Results}

Figure 2 shows the two-dimensional argon ion density in the $1 / 3$ region from the target to the substrate $(\mathrm{y}=0 \sim 0.02 \mathrm{~m})$. The selected region has high plasma density confined by a magnetic field $(x=0.043 \sim 0.128 \mathrm{~m})$. The reason for the profile of the argon ion density is that the magnetized electrons having the Larmor radius $\left(\mathrm{r}_{\mathrm{L}}=0.3 \mathrm{~mm}\right.$ when the electron temperature is $3 \mathrm{eV}$ ) which is much

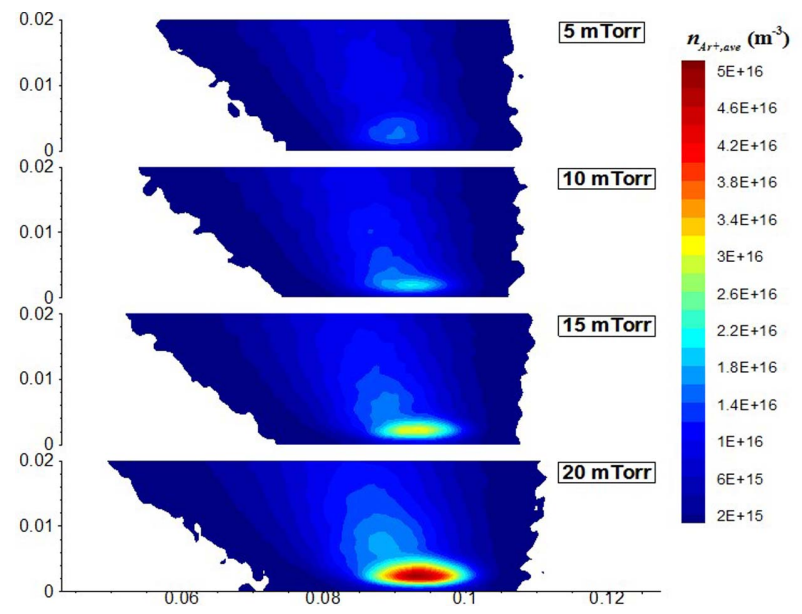

Figure 2. The two-dimensional argon ion density with variation of gas pressure in the $1 / 3$ region from the target to the substrate $(y=0 \sim 0.02 \mathrm{~m}$ and $x=0.043 \sim 0.128 \mathrm{~m})$. The length unit is meter.

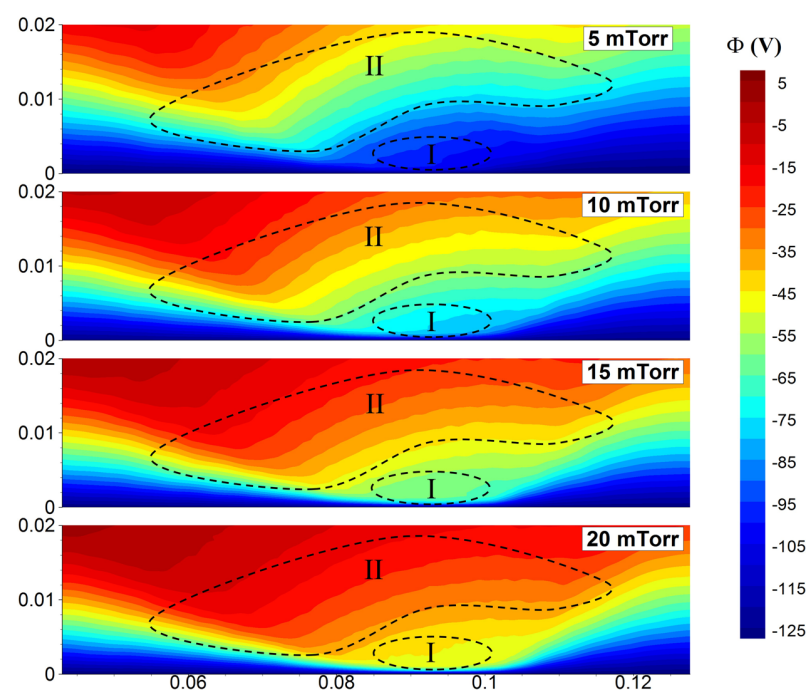

Figure 3. The two-dimensional profile of potential with variation of gas pressure in the $1 / 3$ region from the target to the substrate $(y=0 \sim 0.02 \mathrm{~m}$ and $x=0.043 \sim 0.128 \mathrm{~m})$. The length unit is meter.

smaller than system size. They are confined by the mirror effect. The density profiles of unmagnetized ions are controlled by the ambipolar diffusion and the electron 


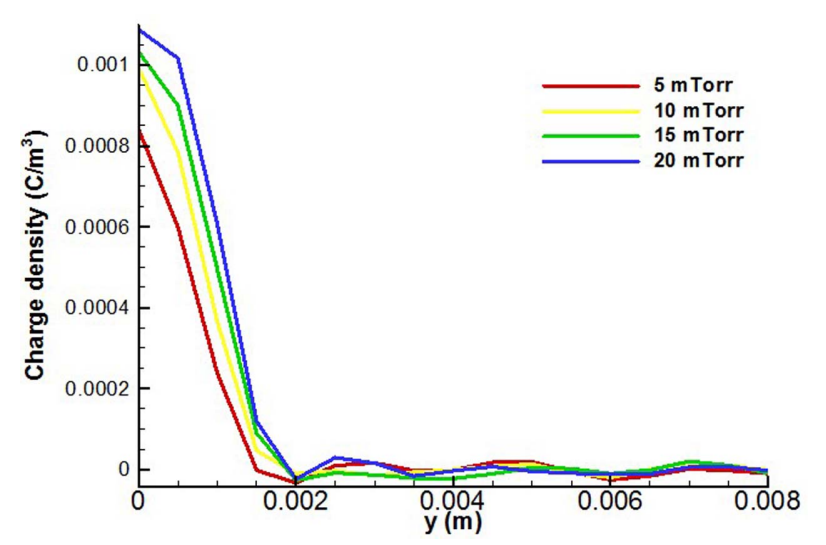

Figure 4. The space charged density for the variation of gas pressure in the sheath region.
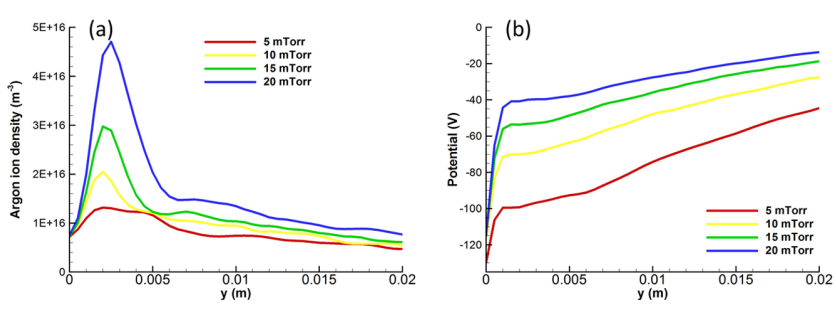

Figure 5. The one-dimensional profile of (a) the argon ion density and (b) potential on the line of $x=0.092$ presented the 1 / 3 region from the target to the substrate $(0 \sim 0.02 \mathrm{~m})$.

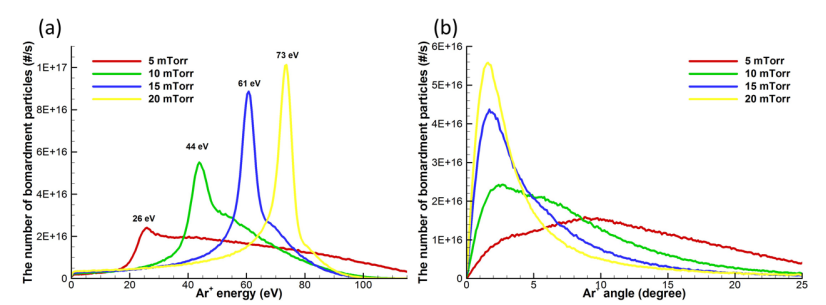

Figure 6. (a) the ion energy distribution function and (b) the ion angle distribution function for bombarding ions.

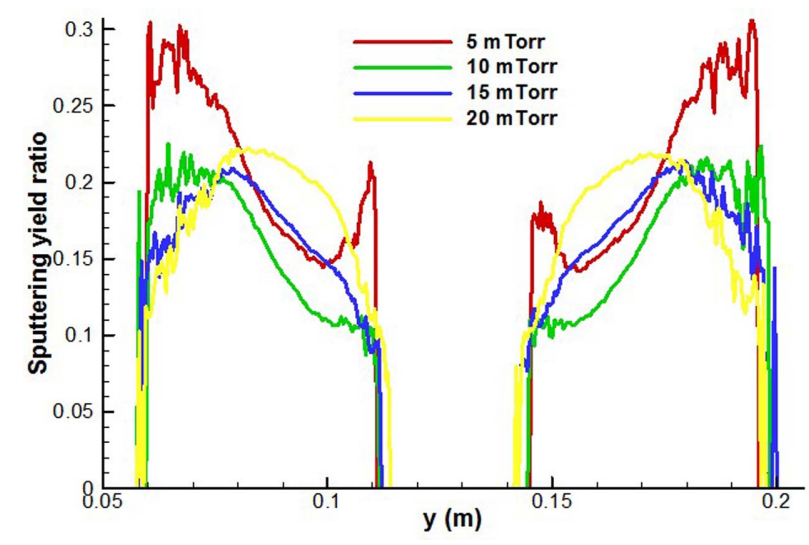

Figure 7. The sputtering yield ratio of the copper target for bombarding the argon ion with the variation of gas pressure.

impact ionization. As a result, the plasma density is high at $\mathrm{x}=0.092 \mathrm{~m}$. The other position where the plasma density is high is at $\mathrm{x}=0.164 \mathrm{~m}$. Also, the plasma density increases with the increase of the gas pressure because of the high ionization rate which increases in proportion to the neutral density.

Figure 3 shows the profile of potential in the same region of Fig. 2. The potential profile is directly related to the space charge. The space charge density becomes high in the sheath region as the gas pressure increases because of high plasma density and electron transport. The transport of magnetized electrons is related to the collision frequency, which is hindered in the direction of the electric field at low pressure. The electron transport in the $+y$ direction is high at high pressure. Therefore, the space charge density is higher for higher gas pressure because of relatively low electron density in the sheath region as shown in Fig. 4.

Figure 5 shows the profiles of argon ion density and plasma potential on the line of $x=0.092$. It represents the $1 /$ 6 region from the target to the substrate $(0 \sim 0.01 \mathrm{~m})$. The potentials on the target electrode are not the same because the simulation was conducted on the current-driven mode. The electrode potential on the target for 10, 15, 20 mTorr cases are about $-117 \mathrm{~V}$, but that for $5 \mathrm{mTorr}$ is about $130 \mathrm{~V}$. The current driven simulations of the same source current require the same ion current to the target. If the target potential is fixed to be the same in the voltage-driven mode, the ion current decreases with the decrease of gas pressure because the ion density also decreases.

Figure 6(a) shows the ion energy distribution functions (IEDFs) for the ions bombarding the target. The most probable bombarding energy of 5, 10, 15, and 20 mTorr cases are 26, 44, 61, and $73 \mathrm{eV}$, respectively. These energies are the same as the voltage differences between the target electrode and the points where the plasma density is maximum as shown in Fig. 5 and region I of Fig. 3. Differently from the nonmagnetized plasmas, the IEDF of low pressure $(5 \mathrm{mTorr})$ is wider than that of 10,15 , 20 mTorr. The ions having a higher energy than the most probable bombarding energy comes from region II of Fig. 3. The mean free path of argon ions at a pressure of $5 \mathrm{mTorr}$ is $9.2 \sim 13.2 \mathrm{~mm}$ if the ion energy ranges between 10 and $100 \mathrm{eV}$. It means that the argon ions generated in region II of Fig. 3 by electron impact ionization are accelerated toward the target and experience collisions once or twice. On the other hand, the mean free path of argon ions at 20 mTorr is $2.3 \sim 3.3 \mathrm{~mm}$ if the ion energy ranges between 10 and $100 \mathrm{eV}$. It means that the accelerated ions experience collisions 5 10 times and lose their energy by collisions at high pressure. Therefore, the number of high energy ions is large at low pressure, 5 mTorr. The ions having low energy below the most probable bombarding energy come from region I and II of Fig. 3. The sheath length is about $1 \mathrm{~mm}$ which is smaller than the mean free path of argon ion at 20 mTorr. It means that the argon ions in region I are accelerated toward the target without collisions. Therefore, the number of low energy ions is small compared with the number of ions having the most probable energy at every pressure. 
Figure 6(b) shows the ion angle distribution functions (IADFs) for bombarding ion to the target. The most probable incident angle increases (that is to say, the IADFs become wide) as the gas pressure decreases because of the same reason of collected high energy ions. The ions having low incident angle come from region I in Fig. 3 perpendicular to the target for every pressure case.

Figure 7 shows the sputtering yield ratio on the copper target from the bombarding argon ions. The sputtering yield ratio increases with the increase of gas pressure from $10 \mathrm{mTorr}$ to $20 \mathrm{mTorr}$ because of the high ion bombarding energy in high pressure. The sputtering yield ratio of 5 mTorr is high in spite of low sheath potential drop because ions with a large incident angle and a high energy come from region II. The yield ratio increases by $20 \sim 30 \%$ compared with the normal incidence when the incident angle is 30 degree [23]. The number of ions over $80 \mathrm{eV}$ is larger for $5 \mathrm{mTorr}$ than those of high pressure as shown in Fig. 6(a).

\section{Conclusions}

In this study, we report the two-dimensional particle-incell simulation of a DC MS system with the variation of gas pressure. The simulation results show the plasma density and potential profiles, and the energy and the angle distributions of the ions incident on the target. It was found out that there are two different mechanisms to control the incident angles of the ions on the target at low pressure. Even though the gas pressure is very low enough to allow ion mean free paths larger than the sheath size, the ion energy and angle distribution can be very wide by the change of plasma potential distribution. It is because the electron transport is hindered at low pressure in the magnetized plasma because of few collisions while the ions are not magnetized. Therefore, the space charge effect becomes more dominant in low pressure MS discharges, and thus the potential profiles make the incident angles wider. The most probable ion energy is determined by the potential difference between the target and the position of peak plasma density rather than the sheath potential. The variation of IEDF and IADF results in significant changes in sputtering yield from the target even with the fixed magnetic field profiles.

\section{Acknowledgments}

This work was supported by a 2-Year Research Grant of Pusan National University and by BK21PLUS Creative Human Resource Development Program for IT Convergence.

\section{References}

[1] P. J. Kelly, and R. D. Arnell, Vaccum 56, 159-172 (2000).

[2] W. Gao and Z. Li, Ceram. Int. 30, 1155-1159 (2004).

[3] K. Sarakinos, J. Alami, and S. Konstantinidis, Surf. Coat. Tech. 204, 1661-1684 (2010).

[4] K. Ellmer and T. Welzel, J. Mater. Res. 27, 765-779 (2012).

[5] E. M. Park, D. H. Lee, and M. S. Suh, Appl. Sci. Converge. Technol. 25, 128-132 (2016).

[6] H. Ahn, D. Lee and Y. Um, Appl. Sci. Converge. Technol. 26, 1115 (2017).

[7] S. H. Jeong and J. H. Boo, Thin Solid Films 447-448, 105-110 (2004)

[8] S. Mraz and J. M. Schneider, J. Appl. Phys. 100, 023503 (2006).

[9] M.-J. Keum and J.-H. Han J. Korean Phys. Soc. 53, 1580-1583 (2008).

[10] H. C. Nguyen, T. T. Trinh, T. Le, C. V. Tran, T. Tran, H. Park, and V. A. Dao, J. Yi, Semicond. Sci. Technol. 26, 105022 (2011).

[11] J. P. Verboncoeur, Plasma, Phys. Controlled Fusion 47, A231 (2005).

[12] C. H. Shon, J. K. Lee, H. J. Lee, Y. Yang, and T. H. Chung, IEEE T. Plasma Sci. 26, 1635-1644 (1998).

[13] C. H. Shon and J. K. Lee, Appl. Surf. Sci. 192, 258-269 (2002).

[14] S. Kuroiwa, T. Mine, T. Yakisawa, T. Makabe, J. Vac. Sci. Technol. B 23, 2218-2221 (2005).

[15] I. Kolev, A. Bogaerts, IEEE T. Plasma Sci. 34, 886-894 (2006).

[16] T. Makabe, T. Yakisawa, Mater. Sci. Forum 555, 65-71 (2007).

[17] Z. Hua-Yu, M. Zong-Xin, Chinese Phys. B 17, 1475-1479 (2008).

[18] V. K. Decyk. T. V. Singh, Comput. Phys. Comm. 185, 708-719 (2014).

[19] C. K. Birdsall, A. b. Langdon, Plasma Physics vis Computer Simulation, Taylor \& Francis Group (2005)

[20] V. Vahedi, M. Surendra, Comput. Phys. Comm. 87, 179-198 (1995).

[21] P. Sigmund, Phys. Rev. 184, 383-416 (1969).

[22] M. P. Seah, T. S. Nunney, J. Phys. D: Appl. Phys. 43, 253001 (2010).

[23] R. Behrisch and W. Eckstein, Sputtering by particle Bombardment, Springer (2007)

[24] Y. Yamamura, H. Tawara, Atom. Data Nucl. Data 62, 149-253 (1996).

[25] V. Vahedi, G. DiPeso, J. Comput. Phys. 131, 149-163 (1997). 